\title{
Envolvente del Centro de Arte Botín en Santander. Estudio de viabilidad de la solución con discos cerámicos roscados sobre chapa de aluminio
}

\section{Building envelope of the Botín Art Centre in Santander. Viability study of the solution using ceramic discs screwed onto aluminium sheet}

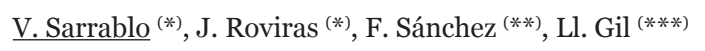

\section{RESUMEN}

El Centro de Arte Botín de Santander presenta una envolvente compleja geométricamente y sofisticada constructivamente de la que destaca su última capa cerámica de reflejos nacarados. Esta capa consiste en un novedoso sistema de anclaje de discos cerámicos a una bandeja de aluminio mediante varilla roscada fijada con resina epoxi. En este artículo se tratan los aspectos de desarrollo técnico y montaje en obra de este cerramiento continuo y se muestran algunos de los resultados de la campaña de ensayos realizada para demostrar la viabilidad de esta capa ante los requerimientos del clima marítimo en Santander.

Palabras clave: envolvente cerámica; discos cerámicos; discos cerámicos roscados; gres porcelánico esmaltado; esmalte nacarado.

\section{ABSTRACT}

The Botin Art Centre in Santander has a geometrically complex and constructively sophisticated architectural envelope. What stands out is its outer pearly sheen ceramic cladding. It consists of an innovative disc-shaped ceramic anchoring system attached to an aluminium tray by means of a threaded rod fixed with epoxy resin. In this article, we will address the technical development and on-site set up of this continuous enclosure. We will also comment on the results of the test runs performed to prove the viability of this cladding in Santander's maritime climate.

Keywords: ceramic envelope; ceramic discs; threaded ceramic discs; glazed porcelain tiles; pearly enamel.

(*) Universitat Internacional de Catalunya. Barcelona (España).

(**) Disset, S. L. Mataró, Barcelona (España).

(***) Universitat Politècnica de Catalunya. Barcelona (España).

Persona de contacto/Corresponding author: sarrablo@uic.es (V. Sarrablo).

Cómo citar este artículo/Citation: Sarrablo, V., Roviras, J., Sánchez, F., Gil, Ll. (2016). Envolvente del Centro de Arte Botín en Santander. Estudio de viabilidad de la solución con discos cerámicos roscados sobre chapa de aluminio. Informes de la Construcción, 68(544): e165, doi: http://dx.doi.org/10.3989/ic.16.002.m15.

Copyright: (C) 2016 CSIC. Licencia / License: Salvo indicación contraria, todos los contenidos de la edición electrónica de Informes de la Construcción se distribuyen bajo una licencia de uso y distribución Creative Commons Attribution License (CC BY) Spain 3.o. 


\section{INTRODUCCIÓN}

El Centro Botín de Arte y Cultura, de unos 6.00o metros cuadrados y diseñado por el estudio Renzo Piano Building Workshop en colaboración con Luis Vidal Arquitectos, se prevé como uno de los edificios más emblemáticos de España durante los próximos años. Se propone como objetivo crear un espacio para el arte, la cultura y la actividad formativa y generar un nuevo lugar de encuentro en Santander que acerque el centro de la ciudad a la bahía, en una operación urbanística que devuelva el mar a la ciudad. El proyecto, levitado sobre los Jardines de Pereda, cuenta con una galería de exposiciones, una zona para actividades educativas y un auditorio de 300 plazas y se divide en dos volúmenes lobulados de diferentes tamaños elevados sobre columnas para liberar el suelo al jardín y a la ciudad y volados parcialmente sobre el mar para reflejar su brillo y movimientos (1), tal como se presenta en la Figura 1.

Uno de los elementos destacados de su volumetría es su innovadora envolvente de acabado cerámico que propone una superficie continua entre las cubiertas, las fachadas y los techos sobre el parque en el que se suspenden, siguiendo una geometría que en algunas zonas presenta doble curvatura (Figura 1). El peculiar diseño de estas envolventes, recubiertas con 280.000 discos de gres abombados y esmaltados en un color nacarado, evoca a un vestido ornamentado con perlas.

Para conseguir el desarrollo técnico de este novedoso cerramiento, llamado Materia CAB Disset, ha sido necesario un proyecto de investigación liderado por la empresa Disset que le ha permitido ser el único agente industrial capaz de diseñar, producir y ejecutar todo el sistema resolviendo cada uno de los aspectos arquitectónicos, técnicos y de instalación requeridos. Para ello ha utilizado procesos de modelado $3 \mathrm{D}$, discretizaciones de formas complejas para la fabricación industrial, diseños paramétricos con procesos industriales procedentes de industrias como la naval o la del automóvil y procesos artesanales imbricados con grandes producciones en serie.

En colaboración con Disset, la Cátedra Cerámica de Barcelona de la Universitat Internacional de Catalunya ha realizado el estudio de viabilidad de la envolvente mediante una campaña de ensayos que ha respondido a los exigentes requerimientos técnicos que demanda el cerramiento de un edificio situado frente al mar Cantábrico.

\section{CERRAMIENTO Y SISTEMA MATERIA CAB DISSET}

Aunque existe una amplia bibliografía sobre envolventes cerámicas resulta complicado encontrar textos que traten sobre revestimientos en formatos de discos circulares y menos aún hallar ejemplos de baldosas roscadas al soporte. Si queremos encontrar un referente cercano al del Centro de Arte Botín tendríamos que recurrir a otro edificio anterior del mismo arquitecto, el aeropuerto de Kansai, que se cierra con una piel metálica también drenante de doble curvatura que oculta la impermeabilización. Este tipo de soluciones reduce el ensuciamiento de la envolvente continua puesto que el agua que arrastra el polvo acumulado en la superficie discurre por la cámara. En estas envolventes continuas no estancas su principal dificultad reside en la fijación de la capa superior al soporte sin interrumpir la impermeabilización (2).
Un ejemplo aún más próximo, esta vez con recubrimiento cerámico, es la Escuela Universitaria de Magisterio en Granada del arquitecto Ramón Fernández-Alonso. En esta edificación también el recubrimiento cerámico de perfiles extrudidos es una envolvente continua que gravita sobre el suelo y debe sujetarse a una estructura de soporte sin interrumpir la capa impermeable que ofrece una chapa de acero (3). Pero quizás el referente más cercano, que también presenta doble curvatura, sea la envolvente no estanca de escamas cerámicas de la Villa Nurbs que soluciona la sujeción al soporte colgando las piezas sobre una red de cables de acero distanciados por unos discos de goma de la lámina impermeable de caucho EPDM, situada protegida detrás (4).

Centrándonos ahora en el Centro de Arte Botín, la primera capa del cerramiento sobre la estructura principal recurre a una chapa base de acero grecada que cierra los espacios entre vigas y sirve de base para la barrera de vapor, el aislamiento térmico de $140 \mathrm{~mm}$ y los clips de poliamida para fijar la chapa perfilada de aluminio que recubre al aislamiento como capa de impermeabilización. Hasta aquí el sistema de cubrición está homologado y tiene una amplia experiencia de uso.

Sobre esta impermeabilización se coloca el sistema de envolvente Materia $C A B$ Disset que es nuevo en el mercado y se ha diseñado específicamente para este edificio. Presenta las siguientes capas según su orden de colocación (Figura 2):

1. Una subestructura de nivelación.

2. Un recubrimiento continuo de bandejas nervadas de aluminio.

3. 280.00o discos cerámicos fijados a las bandejas anteriores mediante varillas roscadas.

En primer lugar se precisa una subestructura de nivelación, mediante directrices rectas y curvas, que define la geometría básica de toda la envolvente y que se instala sobre el sistema de impermeabilización ya ejecutado mediante unas grapas soporte de aluminio. Estas grapas unidas mecánicamente a los nervios de las bandejas de impermeabilización cada $336 \mathrm{~mm}$ fijan las vigas de nivelación. Éstas consisten en dos perfiles de aluminio en $\mathrm{U}$, uno inferior en fragmentos fijados cada $850 \mathrm{~mm}$ a las nervaduras de la chapa mediante grapa y otro superior continuo que fija y regula la posición de la bandeja de remate de la envolvente. Estos dos perfiles se solapan con una unión dentada (Figura 3) que posibilita el movimiento longitudinal de la parte superior debido a acciones térmicas a la vez que facilita la nivelación (Figura 4). Una vez conseguido el ajuste geométrico requerido se remachan puntualmente.

A continuación de la subestructura de nivelación se fijan sobre ésta las bandejas de aluminio nervadas, planas o curvas según su posición en el edificio, que poseen un lacado especial para ambientes agresivos como el marino (con sello Qualicoat Seaside, que garantiza su protección y durabilidad). Las nervaduras de estos elementos se definen en sección para soportar las cargas de peso propio del sistema, sobrecarga de viento, nieve y uso (en las cubiertas) y transmitirlas directamente a la subestructura de nivelación.

Para el correcto despiece y modulación de la envolvente, las bandejas se suministran en obra numeradas, cortadas en taller y con los taladros con rosca ya situados para la colocación de los discos cerámicos (Figura 5). 

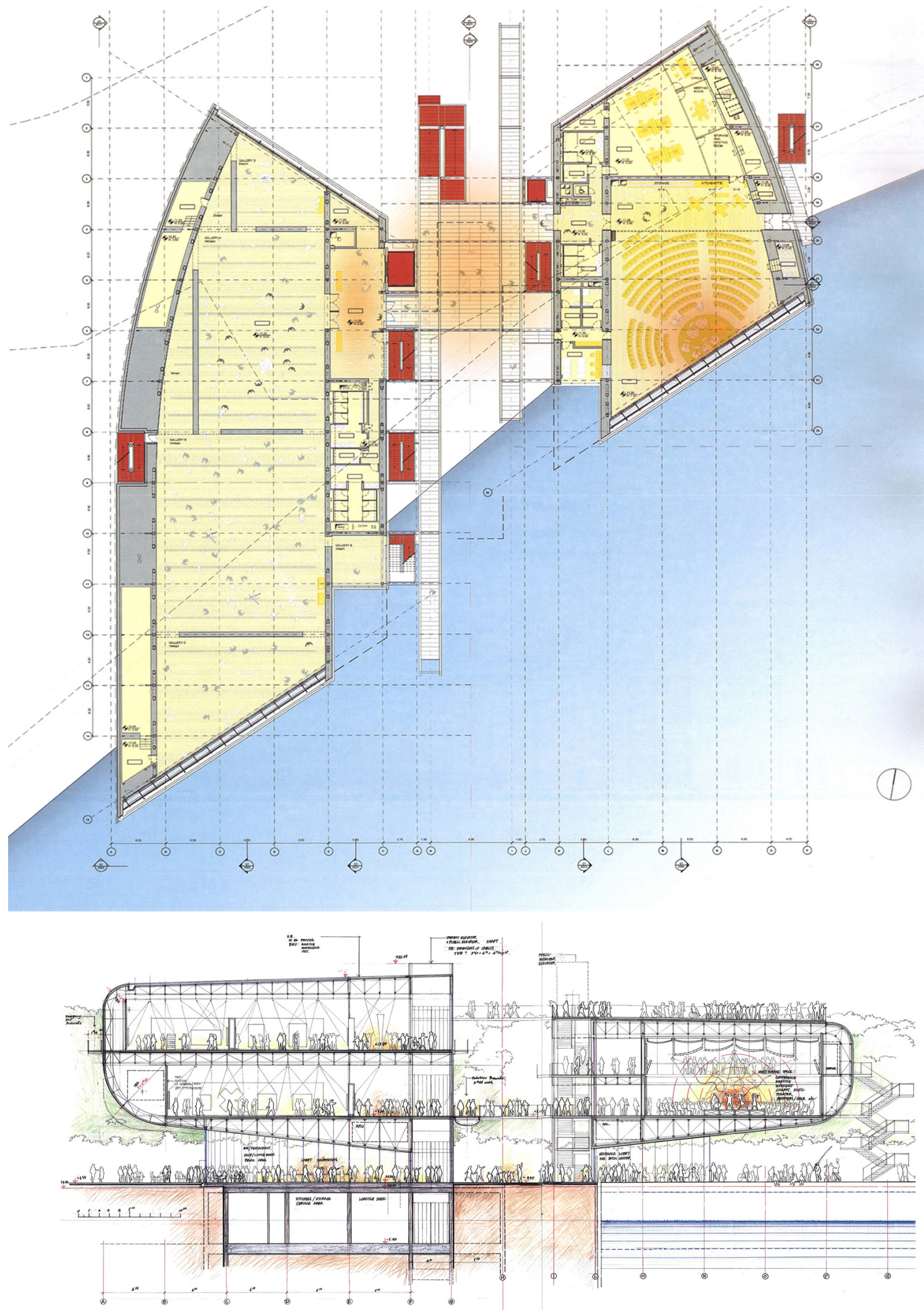

Figura 1. Planta + sección general y sección de detalle (Archivo Renzo Piano Building Workshop). 

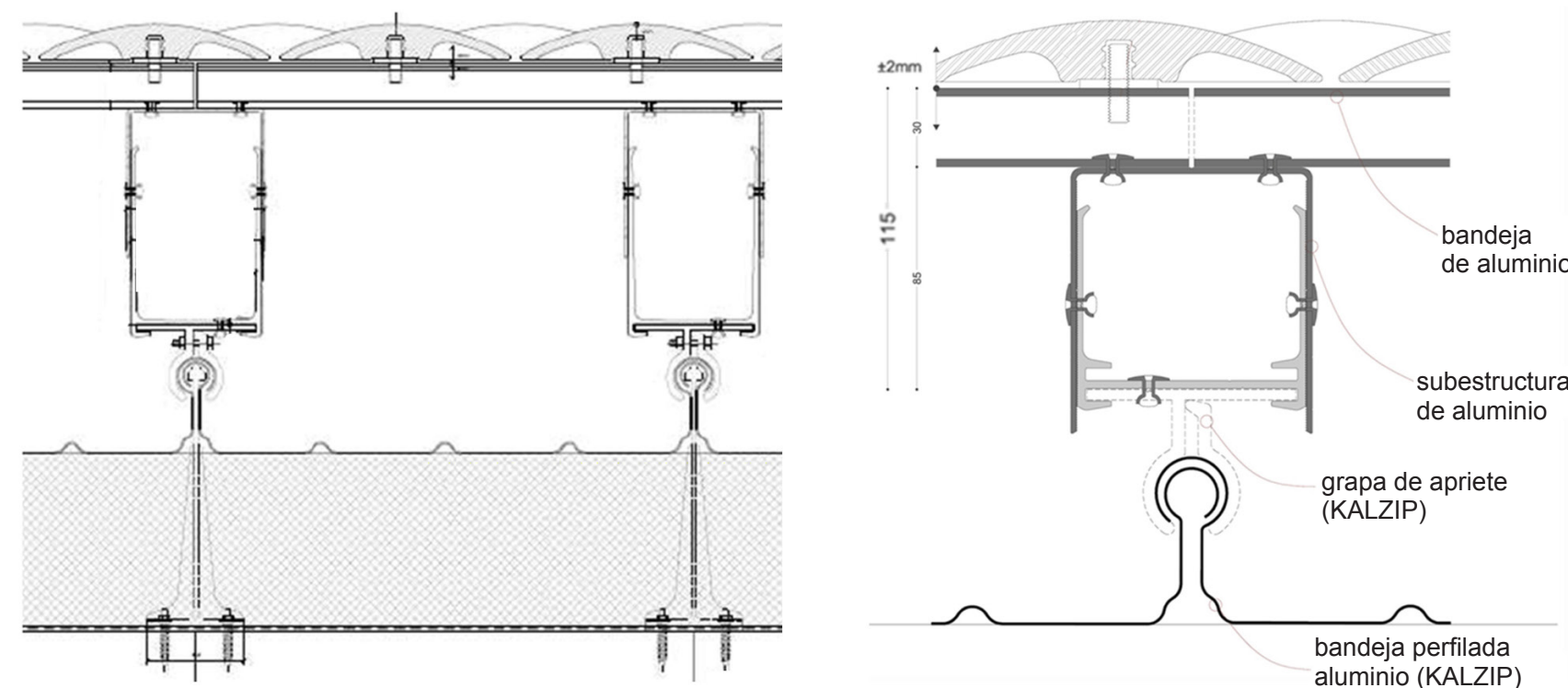

Figura 2. Sección tipo del sistema Materia CAB Disset sobre la chapa de impermeabilización (Archivo Disset).

Por último, se anclan los discos cerámicos. Estas piezas, producidas por Cerámica Cumella, están realizadas con una pasta de gres de alta temperatura (cocción a $1.250^{\circ} \mathrm{C}$ ) de una absorción de agua inferior al $2 \%$ y se obtienen por centrifugado ${ }^{1}$, formando discos abombados de $21 \mathrm{~mm}$ de altura y $156 \mathrm{~mm}$ de diámetro. Se esmaltan en su parte convexa con un esmalte feldespático brillante, de color blanco cálido y se cuecen en horno en una atmósfera oxidante-neutra a alta temperatura que les proporciona una excelente resistencia al choque térmico, al cuarteo y al hielo, así como una total inalterabilidad ante ácidos y álcalis. En una segunda cocción se aplica un esmalte nacarado que les aporta su característico aspecto de fragmento de perla (Figura 7). Este tipo de acabado irisado ya se ha ensayado con éxito en el auditorio de Algueña (Murcia), de COR Asociados (5).

En su cara posterior estos discos disponen de un alojamiento de $10 \mathrm{~mm}$ de diámetro por $15 \mathrm{~mm}$ de profundidad mecaniza- do en su interior para introducir en taller una varilla roscada adherida con resina epoxi que sirve de anclaje con las bandejas de aluminio. La fijación de estas piezas en las bandejas se realiza mediante un utensilio mecánico que proporciona la misma fuerza de roscado a todas las uniones. Además, las varillas presentan una resina anaeróbica en su contacto con la rosca de la bandeja (Figura 6) para garantizar que no se producirán desenroscados eventuales, tan sólo permitirá los realizados con utensilio específico.

La disposición de las cerámicas circulares en la envolvente se realiza siguiendo una disposición en línea recta que mantiene la equidistancia entre ellas en vertical y en diagonal en todas las superficies planas y de curvatura simple (Figura 7).

Sin embargo, la complejidad geométrica de la envolvente cerámica cuando adquiere una superficie de doble curvatura

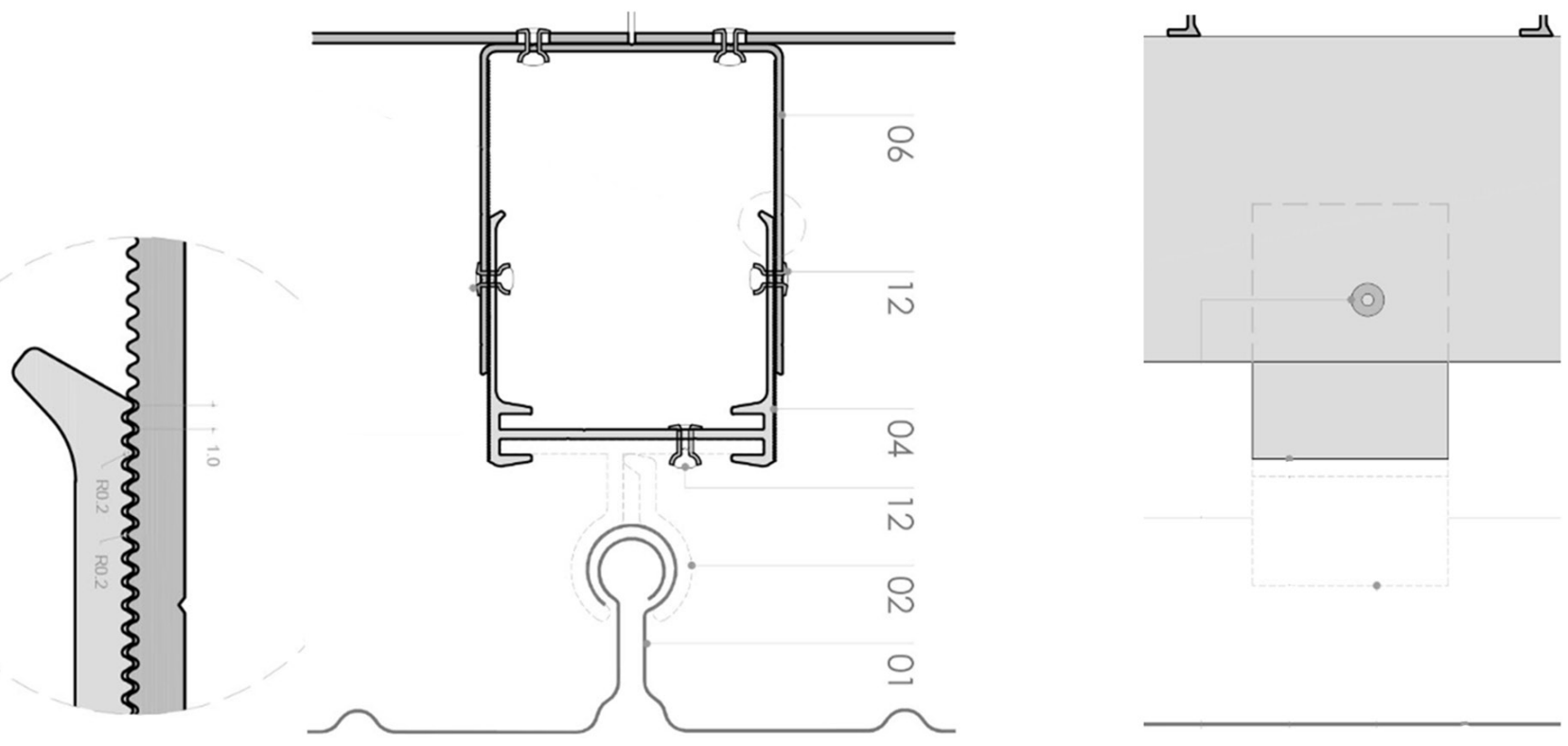

Figura 3. Subestructura de nivelación fijada con grapa sobre la chapa de impermeabilización. Detalle del solape dentado. (Archivo Disset).

\footnotetext{
${ }^{1}$ Se parte de una pasta cerámica blanda que se introduce en un molde rotativo y por revolución se comprimen y conforman las piezas.
} 

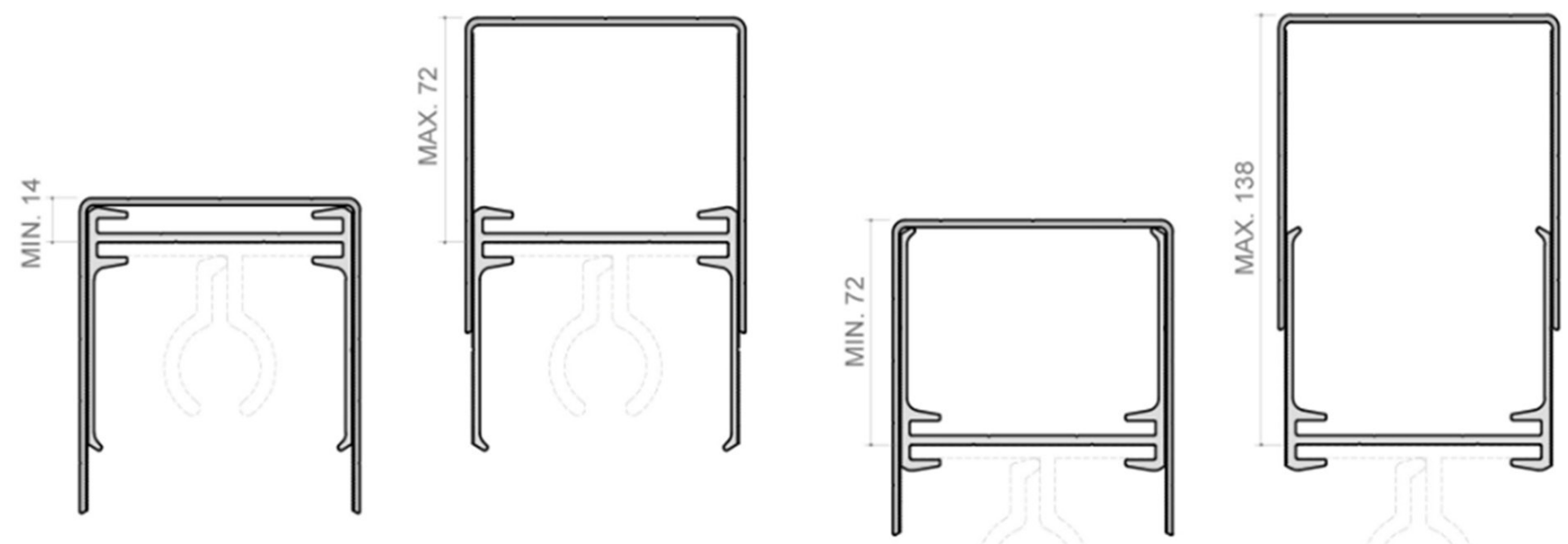

Figura 4. Tolerancias de nivelación de la subestructura (Archivo Disset).
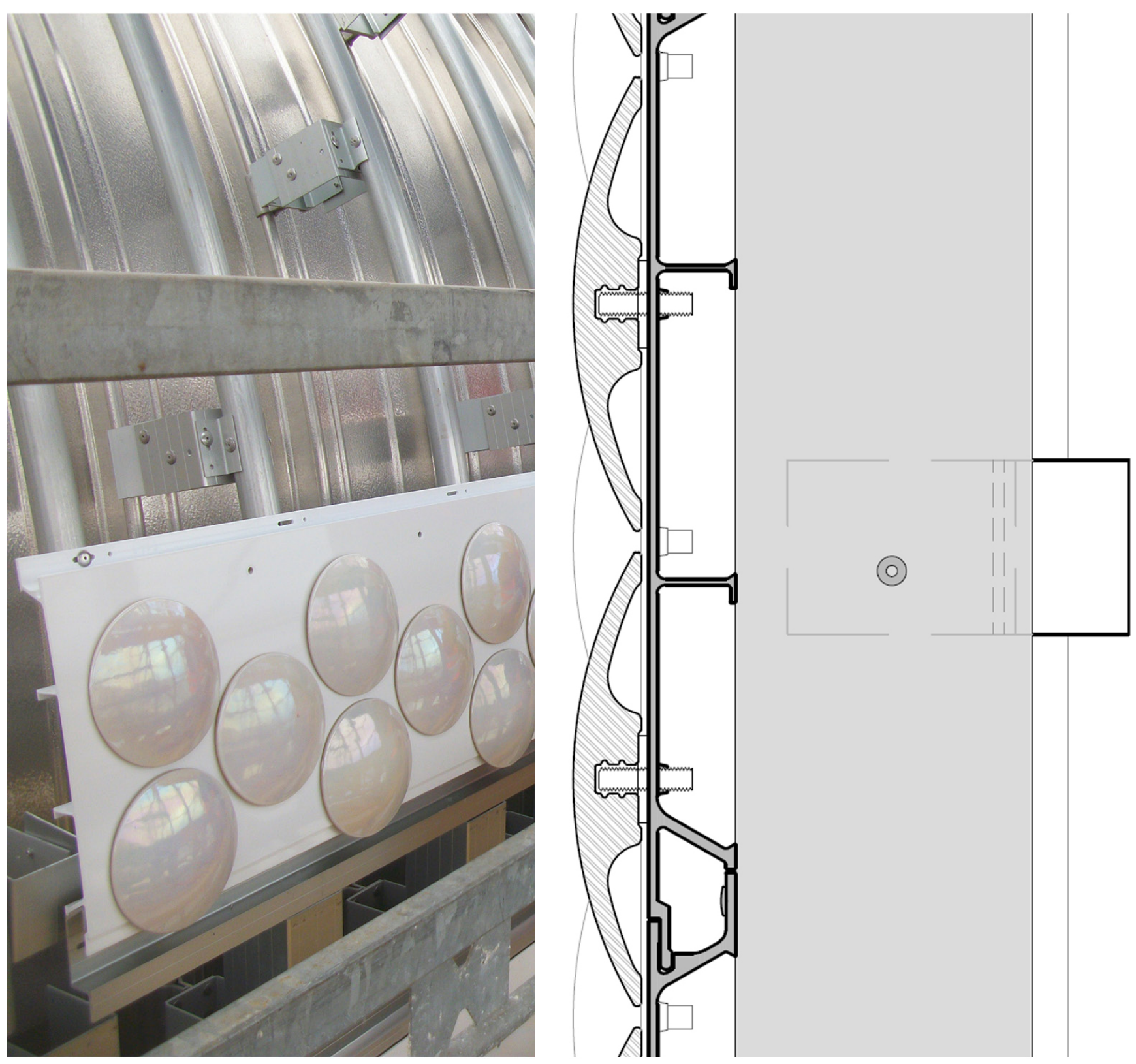

Figura 5. Bandeja de aluminio nervada sobre subestructura de nivelación y discos cerámicos roscados a ella (Archivo Disset). 


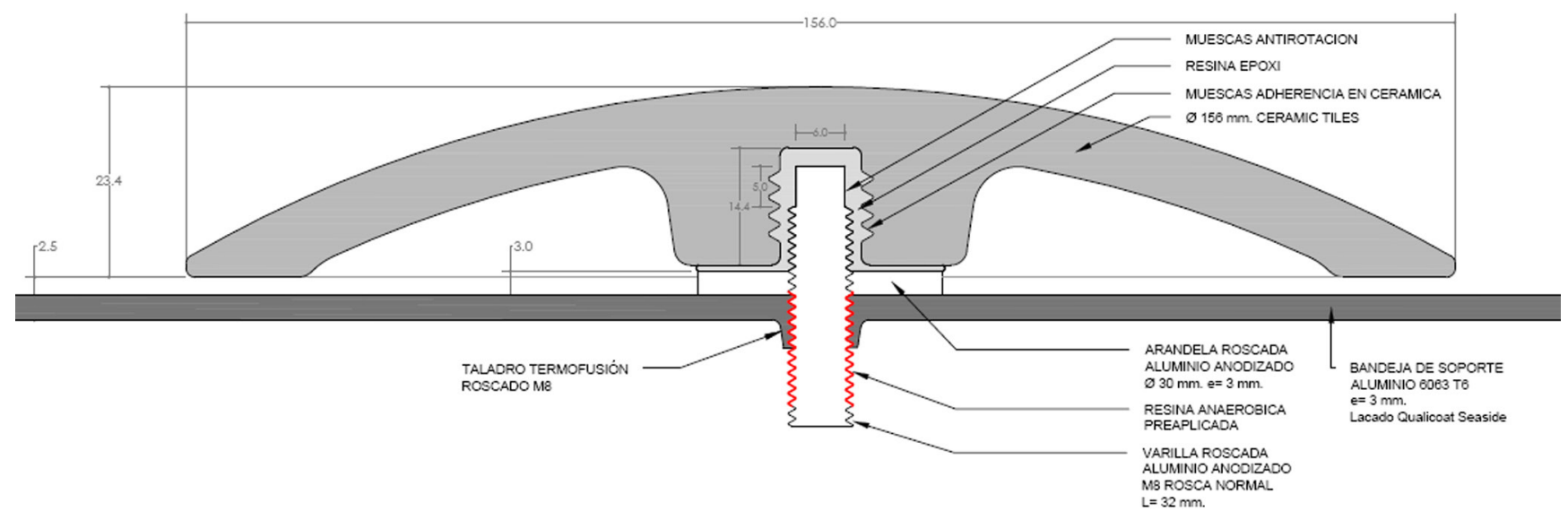

Figura 6. Unión del disco cerámico con la bandeja de aluminio mediante varilla roscada (Archivo Disset).

no desarrollable provoca que las distancias entre piezas cerámicas no sean constantes. Para conseguir una apariencia uniforme se ha desarrollado un sistema de despiece cerámico que permite optimizar los sistemas productivos y de montaje subdividiendo la superficie en zonas seriadas (despiece regular) y no seriadas (despiece irregular) ${ }^{2}$.

El resultado final permite apreciar la ventaja de realizar diseño, fabricación y montaje por un único agente industrial que ha posibilitado ajustar y optimizar cada uno de los requerimientos estéticos, productivos y constructivos en una solución óptima y eficiente para el desarrollo de la obra.

\section{ESTUDIO DE VIABILIDAD TÉCNICA Y CAMPAÑA DE ENSAYOS}

La novedad del sistema Materia $C A B$ Disset ha requerido de un estudio de viabilidad técnica que ofreciera un estricto control de sus respuestas ante acciones mecánicas (resistencia y estabilidad) y de durabilidad (ambiental y fatigas). Este estudio se ha centrado en evaluar las dos zonas que requerían mayores garantías de seguridad: la unión entre el disco cerámico y la varilla metálica roscada y la unión entre esta varilla y la bandeja de aluminio. Para ello se ha realizado una campaña de ensayos en el LITEM (Laboratorio para la Innovación Tec-
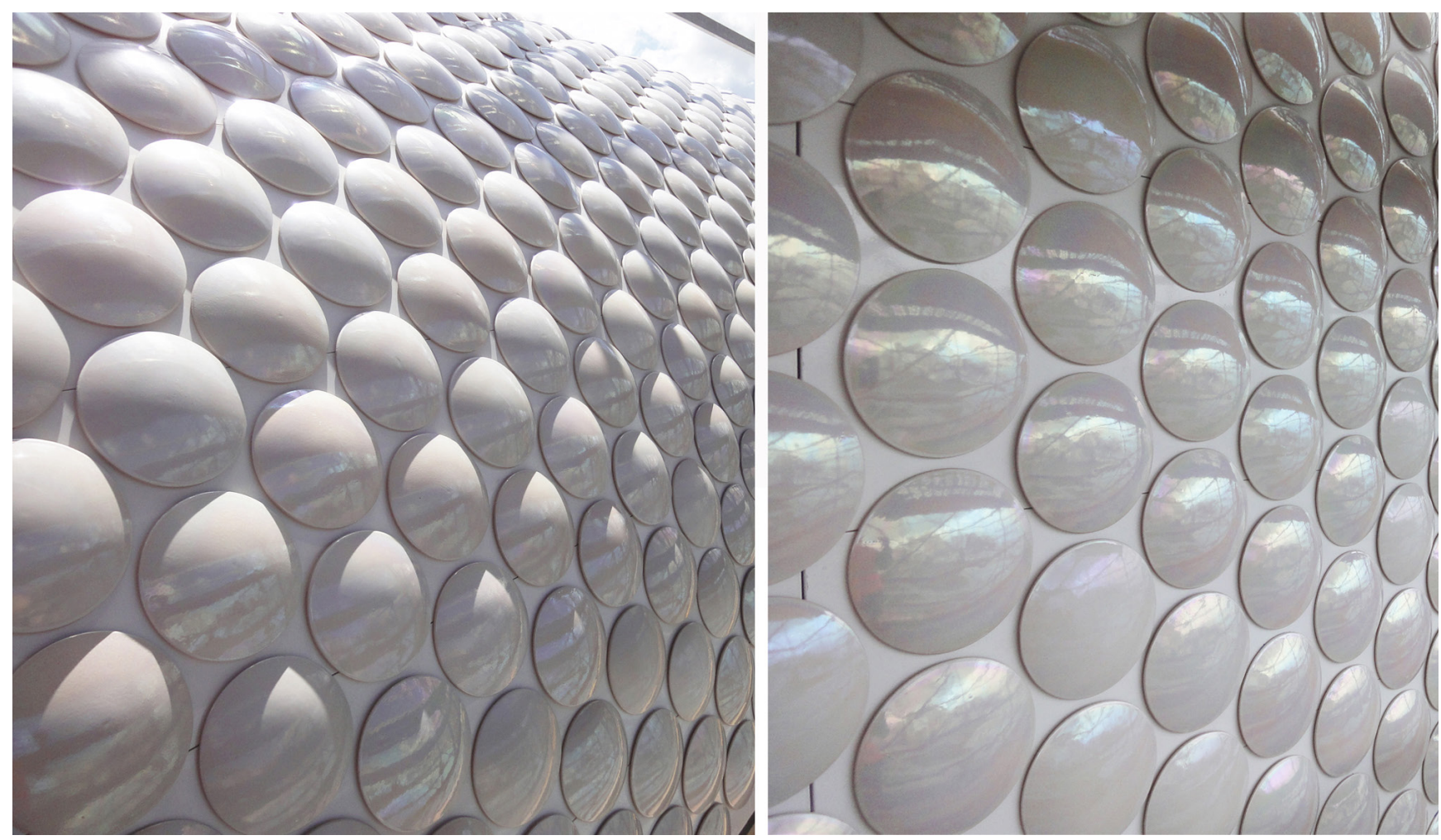

Figura 7. Aspecto de los discos nacarados en superficie curva y plana (Archivo Disset).

\footnotetext{
${ }^{2}$ Se han dibujado la totalidad de discos cerámicos en un modelo $3 \mathrm{D}$, parametrizando las zonas irregulares y desarrollando un software propio de diseño, llamado Recab Disset 20oo, que permite pasar la geometría compleja del 3D a la fabricación y producción de las bandejas en un proceso totalmente automatizado.
} 
nológica de Estructuras y Materiales) de Terrassa, en la que se han ejecutado los siguientes tipos de ensayo:

a) Ensayos de arrancamiento sobre el tornillo de sujeción y su unión con la bandeja de aluminio.

b) Ensayos de arrancamiento sobre el tornillo de sujeción y su unión con la pieza cerámica.

c) Ensayos de par de desapriete.

d) Ensayos de aplicación de carga descentrada en un extremo de la pieza de cerámica.

e) Ensayos de tracción sobre muestras envejecidas de las bandejas de aluminio.

f) Ensayos de vibración inducida de piezas cerámicas roscadas a las bandejas de aluminio.

g) Ensayos de corrosión en cámara de niebla salina de diferentes piezas.

h) Ensayos de hielo/deshielo y choque térmico sobre la pieza de cerámica.

A continuación se detallan los resultados de algunos de ellos, empezando por los requisitos mecánicos.

\subsection{Ensayos de arrancamiento (pull-out) sobre el tornillo de sujeción y su unión con la bandeja de aluminio}

Para determinar la carga de rotura de esta unión se ha procedido a ensayar varios fragmentos de la bandeja con varilla roscada en ella y adherida con una resina anaeróbica bicomponente. En un primer ensayo se ha ejecutado el arrancamiento en rampa continua de carga según el procedimiento 1 de la Figura 8. Posteriormente se han variado la colocación de los utillajes según el procedimiento 2 que se presenta en la misma figura y se han ensayado las muestras con 3 ciclos de carga-descarga de $+/-3,2 \mathrm{KN}$ y hasta rotura con una rampa continua de carga. En un tercer procedimiento se han variado los 3 ciclos de carga-descarga a +/- $200 \mathrm{~N}$ y las probetas se han envejecido previamente en cámara de niebla salina y se han calentado durante el ensayo hasta $60^{\circ} \mathrm{C}$.

Una vez vistos los diferentes resultados (Tabla 1) se comprueba que, aun cambiando la configuración de los útiles de ensayo o los rangos máximos de los ciclos de carga-descarga, la carga máxima no varía excesivamente entre las diferentes
Tabla 1. Resumen de resultados (las probetas con código E corresponden a las muestras envejecidas y calentadas).

\begin{tabular}{|c|c|c|c|}
\hline Probeta & $\begin{array}{c}\text { Carga } \\
\text { máxima (N) }\end{array}$ & Procedimiento & Ciclos \\
\hline o0 & 3.852 & 1 & 1 \\
\hline 01 & 3.518 & 2 & $3[+/-3,2 \mathrm{KN}]$ \\
\hline 02 & 3.269 & 2 & $2[+/-3,2 \mathrm{KN}]$ \\
\hline 03 & 3.334 & 2 & $3[+/-3,2 \mathrm{KN}]$ \\
\hline 07 & 3.292 & 3 & $3[+/-200 \mathrm{~N}]$ \\
\hline 08 & 3.096 & 3 & $3[+/-200 \mathrm{~N}]$ \\
\hline 09 & 3.043 & 3 & $3[+/-200 \mathrm{~N}]$ \\
\hline o6-E & 3.458 & 3 & $3[+/-200 \mathrm{~N}]$ \\
\hline O7-E & 3.122 & 3 & $3[+/-200 \mathrm{~N}]$ \\
\hline 08-E & 3.359 & 3 & $3[+/-200 \mathrm{~N}]$ \\
\hline
\end{tabular}

probetas, que se sitúa entre 3.043 y $3.852 \mathrm{~N}$, con una media de $3.334 \mathrm{~N}$. Tampoco afecta a los resultados la aplicación de una temperatura de $60^{\circ} \mathrm{C}$ y el envejecimiento acelerado de las probetas. En todos los casos la rotura se produce a nivel del roscado de las piezas (Figura 9).

\subsection{Ensayos de arrancamiento (pull-out) entre la varilla roscada y su unión con la pieza cerámica}

En estos ensayos se han vuelto a comparar muestras sin acciones previas y muestras envejecidas y/o calentadas previamente para obtener información sobre la influencia de estos parámetros en la unión mediante resinas epoxi entre la varilla roscada y el disco cerámico. De las quince probetas ensayadas, seis han sido muestras sin acciones previas sobre ellas, tres han sido calentadas previamente mediante foco de infrarrojos hasta $60^{\circ} \mathrm{C}$, tres más calentadas hasta $71^{\circ} \mathrm{C}$ y tres más han sido previamente envejecidas en cámara de niebla salina y posteriormente calentadas hasta $60^{\circ} \mathrm{C}$. Estas muestras se han colocado en útiles similares al ensayo anterior (Figura 10) roscando la varilla en el útil del actuador a tracción y ejecutando el ensayo de pull-out en rampa continua, con carga aplicada mediante control de desplazamiento a una velocidad de 1,25 $\mathrm{mm} / \mathrm{min}$ hasta rotura, que ha ocurrido con fallo abrupto.

Según se muestra en la tabla resumen de resultados, la media de resistencia para las muestras sin acciones previas es 5.749
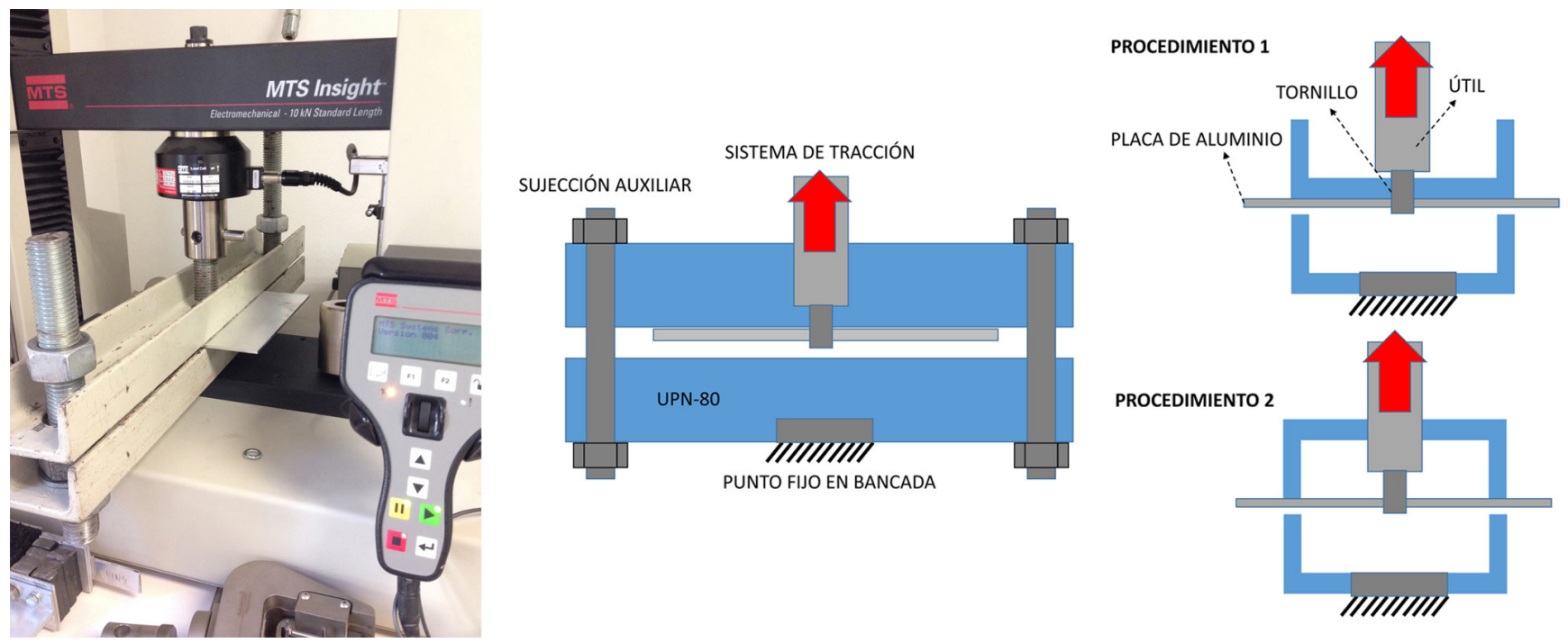

Figura 8. Método de ensayo para el pull-out con los dos procedimientos utilizados (Archivo LITEM). 

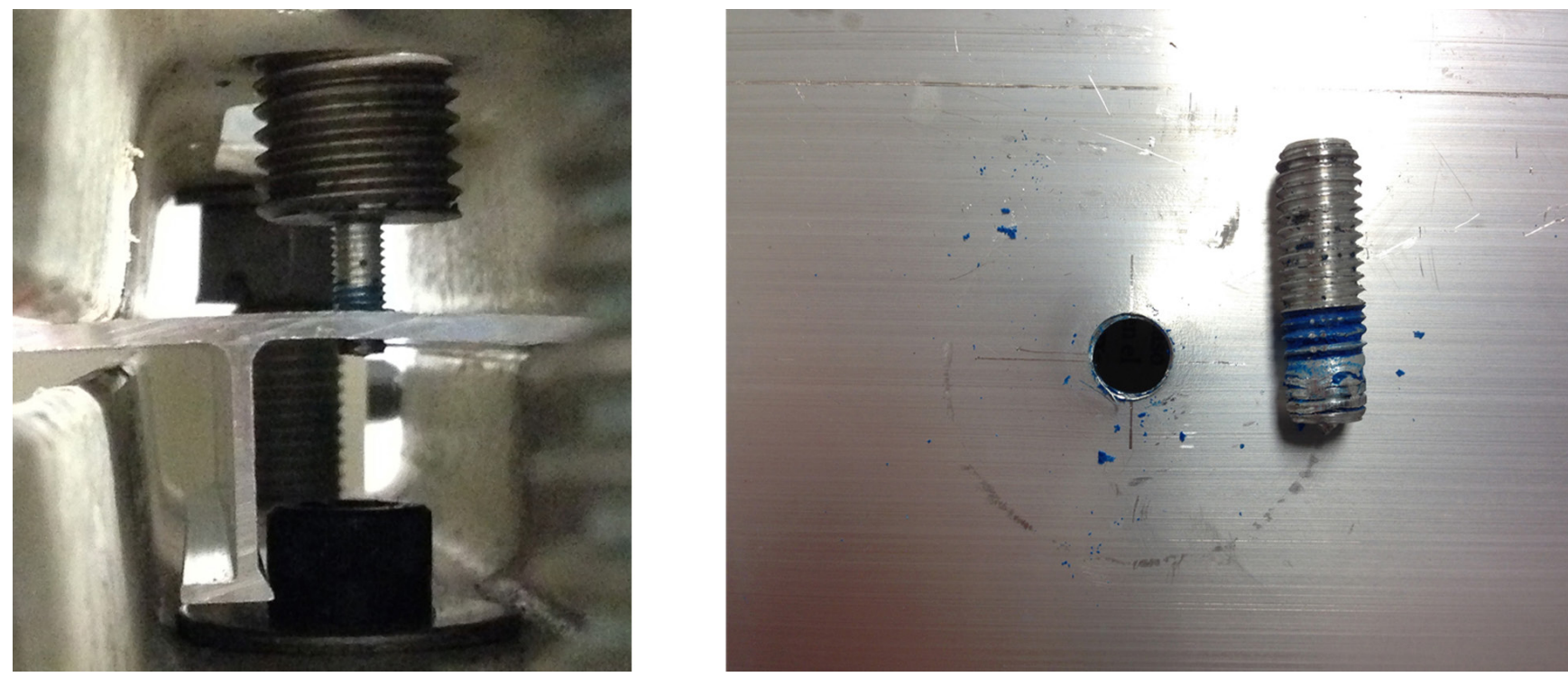

Figura 9. Detalle del estiramiento del tornillo y detalle del fallo en la rosca de la varilla (Archivo LITEM).
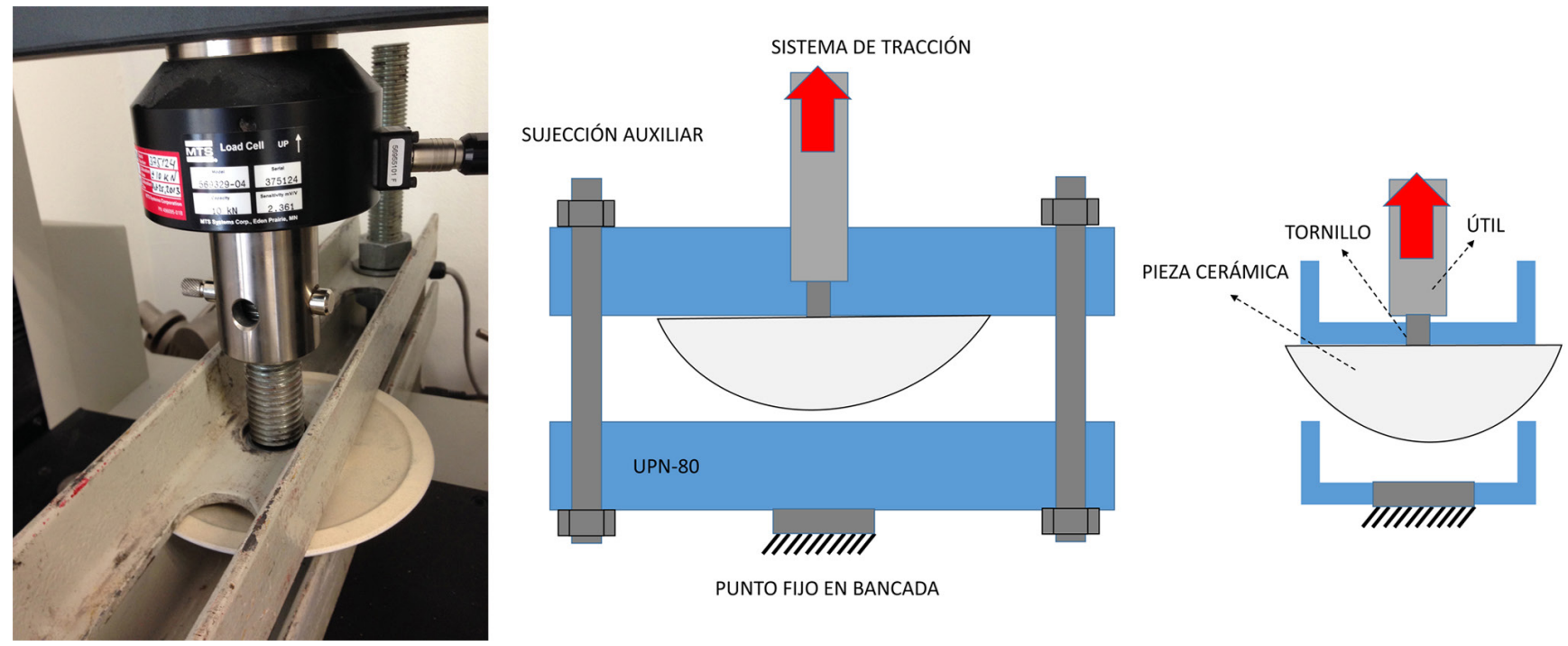

Figura 10. Método de ensayo pull-out entre el disco cerámico y su varilla roscada (Archivo LITEM).

$\mathrm{N}$, mientras que la media para las muestras envejecidas y/o calentadas es de $3.580 \mathrm{~N}$ (no se perciben diferencias notables entre las muestras envejecidas, las muestras calentadas a $60^{\circ} \mathrm{C}$ y las calentadas a $71^{\circ} \mathrm{C}$ ). Por consiguiente, tanto el envejecimiento como el calentamiento inducidos parecen haber afectado a la unión con resinas epoxi de las piezas reduciendo un $38 \%$ su media resistente. De todos modos, salvo la probeta S-21, todas las demás presentan valores ligeramente superiores a los pull-out del ensayo anterior entre varilla y bandeja, por lo que se trasluce que, ante esfuerzos de arrancamiento, resultaría levemente más crítica aquella unión.

En todos los ensayos se ha alcanzado la rotura sin deformación aparente en la muestra y el modo de rotura ha sido catastrófico generalizado con una concentración en la zona de unión del tornillo (Figura 11).

\subsection{Ensayos de par de desapriete}

Además de los ensayos de arrancamiento vistos en los puntos anteriores, también ha resultado preciso determinar el riesgo de aflojamiento de las dos uniones estudiadas debido a un par de desapriete. Se han estudiado 21 muestras con dos tipos diferentes de adhesivos en la unión atornillada: diez de las muestras con adhesivo de resina anaeróbica monocomponente aplicada in situ (roja) y once con resina anaeróbica bicomponente aplicada previamente (azul).

El procedimiento utilizado ha consistido en adherir una arandela en la cabeza de cada pieza cerámica (ya atornillada a un fragmento de bandeja de aluminio) mediante un adhesivo de alta resistencia que soportara altas temperaturas. Una vez curado el adhesivo se han ejecutado los ensayos de par de desapriete con una llave dinamométrica sobre la arandela. Las piezas estaban a temperatura ambiente en doce muestras y se sometieron a $70^{\circ} \mathrm{C}$ en otras nueve.

Las piezas presentaron tres tipos de fallos o roturas:

1. Fallo por la arandela externa para la medición con llave dinamométrica (Figura 12a). Estos fallos se han considerado resultados no válidos.

2. Fallo por la unión adhesiva entre la varilla roscada y el disco cerámico (Figura 12b). 
Tabla 2. Resumen de resultados de los ensayos de pull-out.

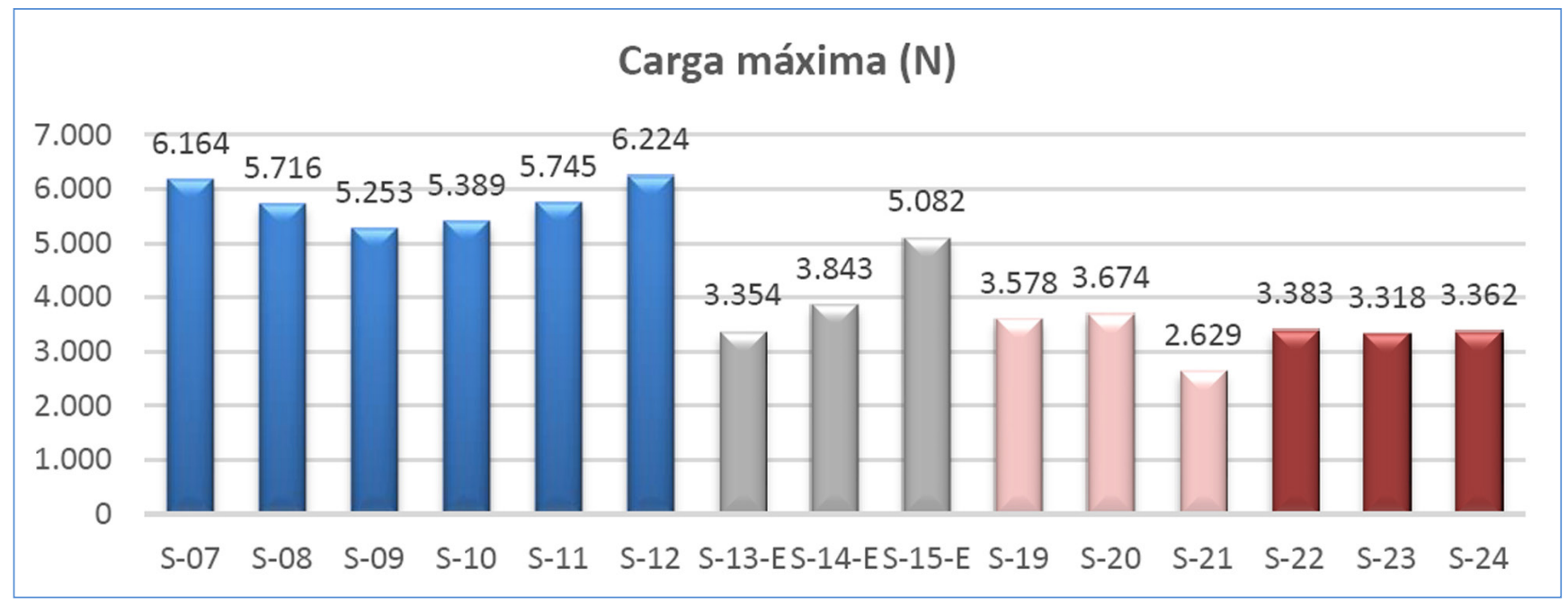

S-07 hasta S-12: Probetas sin acciones previas.

S-13-E hasta S-15-E: Probetas envejecidas y ensayadas con temperatura de $60^{\circ} \mathrm{C}$.

S-19 hasta S-21. Probetas ensayadas con temperatura de $60^{\circ} \mathrm{C}$.

S-22 hasta S-24. Probetas ensayadas con temperatura de $71^{\circ} \mathrm{C}$.

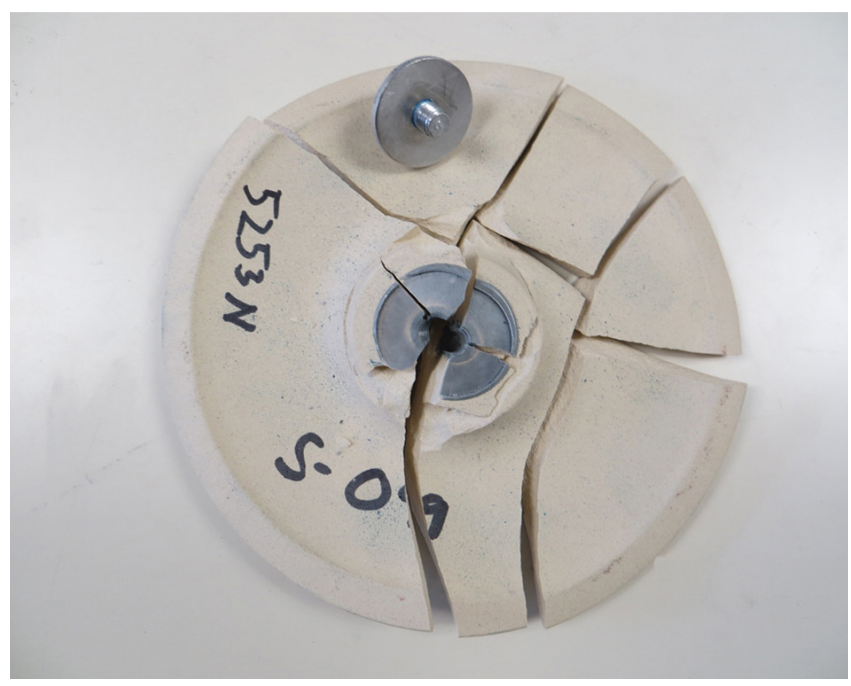

Figura 11. Modo de rotura de las muestras (Archivo LITEM).

3. Fallo por la unión roscada con adhesivo entre la varilla roscada y la chapa de aluminio (Figura 12c).

En algunos ensayos realizados tanto a temperatura ambiente como con calor inducido se han producido errores en el procedimiento cuando han fallado por la arandela externa y, por tanto, no hemos considerado válidos esos resultados. De todos modos, éstos han sido minoría y, aun así, han presentado valores de rotura iguales o superiores al resto que han fallado por las uniones a evaluar y, por tanto, estaban de parte de la seguridad.

De los ensayos considerados válidos, que han sido la mayoría de los realizados (Tablas 3 y 4), se ha podido comprobar que los resultados a temperatura ambiente han proporcionado valores promedio de par de desapriete similares a los ensayos con calor inducido, sobre todo en los que se ha utilizado el adhesivo con resina anaeróbica bicomponente aplicada previamente (azul). Por tanto, se puede concluir que los incrementos de temperatura hasta unos $70^{\circ} \mathrm{C}$ no afectan significativamente a la capacidad de resistencia al desapriete.
Tabla 3. Resultados a temperatura ambiente.

\begin{tabular}{|c|c|c|c|}
\hline Muestra & $\begin{array}{c}\text { Adhesivo } \\
\text { resina }\end{array}$ & Par (Nm) & Tipo de fallo \\
\hline 10 & Roja & 8 & Cerámica/rosca \\
\hline 2 & Roja & $>8$ & Arandela \\
\hline 3 & Roja & $>10$ & Arandela \\
\hline 5 & Roja & 9 & Cerámica/rosca \\
\hline 6 & Roja & 9 & Cerámica/rosca \\
\hline 7 & Roja & $>11$ & Arandela \\
\hline 1 & Roja & $>11$ & Arandela \\
\hline 9 & Azul & 7 & Chapa/rosca \\
\hline 4 & Azul & 7 & Chapa/rosca \\
\hline 1 & Azul & $>8$ & Arandela \\
\hline 2 & Azul & $>7$ & Arandela \\
\hline 3 & Azul & 7 & Chapa/rosca \\
\hline
\end{tabular}

Tabla 4. Resultados con temperatura inducida a $70^{\circ} \mathrm{C}$.

\begin{tabular}{|c|c|c|c|}
\hline Muestra & $\begin{array}{c}\text { Adhesivo } \\
\text { resina }\end{array}$ & Par (Nm) & Tipo de fallo \\
\hline 4 & Roja & 8 & Arandela \\
\hline 8 & Roja & 10 & Cerámica/rosca \\
\hline 9 & Roja & 10 & Arandela \\
\hline 3 & Azul & 6 & Chapa/rosca \\
\hline 5 & Azul & 8 & Cerámica/rosca \\
\hline 6 & Azul & $<4$ & Chapa/rosca \\
\hline 7 & Azul & 6 & Cerámica/rosca \\
\hline 8 & Azul & 11 & Cerámica/rosca \\
\hline 10 & Azul & 7 & Arandela \\
\hline
\end{tabular}

El adhesivo de resina anaeróbica monocomponente aplicada in situ (roja) ha presentado resistencias al par de desapriete ligeramente mayores, con una media $9 \mathrm{Nm}$. Mientras el adhesivo con resina anaeróbica bicomponente aplicada previamente (azul) ha llegado a una media de $7 \mathrm{Nm}$. Las dos medias se consideran resultados correctos para la aplicación que se propone. Todas las roturas con el adhesivo de resina anaeróbica monocomponente aplicada in situ (roja) se dan en la unión entre la varilla roscada y el disco cerámico mientras que con el adhesivo con resina anaeróbica bicomponente aplicada previamente (azul) ocurren la ma- 

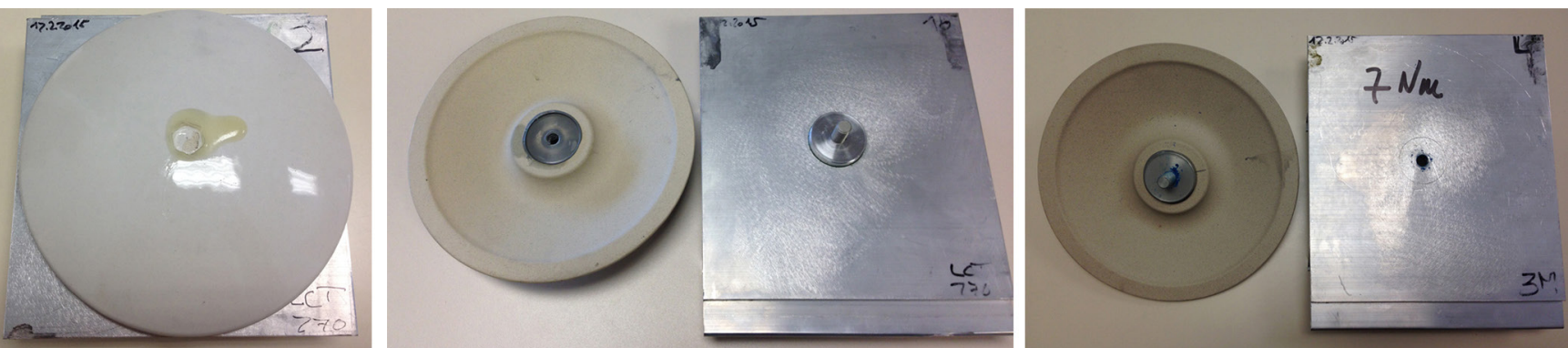

Figura 12. De izquierda a derecha: fallo por la arandela externa, fallo entre la varilla roscada y el disco cerámico y fallo entre la varilla roscada y la bandeja de aluminio (Archivo LITEM).

yor parte de las veces en la unión entre la varilla roscada y la chapa de aluminio.

A continuación se detallan algunos de los ensayos de durabilidad.

\subsection{Ensayos de corrosión en cámara de niebla salina}

Dada la directa exposición de la envolvente frente al mar, se han realizado ensayos de envejecimiento por corrosión en niebla salina de acuerdo con la norma UNE-EN ISO 9227 (6), utilizando una niebla pulverizada con una concentración de un $5 \%$ en peso ( $50 \mathrm{~g}$ de $\mathrm{NaCl} / \mathrm{litro}$ ), un $\mathrm{pH}$ dentro de la cámara entre 6,5 y 7,2 y una temperatura de $35^{\circ} \mathrm{C}$. La duración del ensayo ha sido de cuatro semanas (672 horas), realizándose un control visual de las piezas cada semana.

Se han ensayado cinco muestras de discos cerámicos con varilla roscada incorporada y tres fragmentos de bandejas de aluminio también con varillas roscadas.

Tal como se aprecia en la Figura 13 la arandela metálica que une la varilla al disco cerámico presenta una tonalidad diferente pero sin indicios de corrosión y en las piezas cerámicas no se observa ninguna alteración del material. En las bandejas de aluminio se observa un cambio de tonalidad superficial y la aparición de manchas de diferentes tonalidades, pero tampoco se presentan corrosiones aparentes.

\subsection{Ensayos de hielo/deshielo y choque térmico sobre el disco cerámico con varilla roscada}

La unión entre la pieza cerámica y el tornillo se realiza con un adhesivo tipo epoxi. Sabiendo que la temperatura puede afectar a la capacidad portante del adhesivo se han realizado ciclos de hielo/deshielo y de choque térmico para evaluar

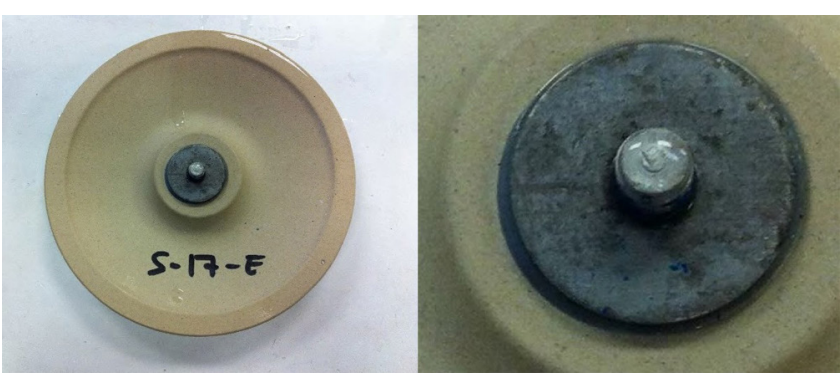

Figura 13. Parte interna del disco cerámico y detalle de la varilla y la arandela (Archivo LITEM). posteriormente, mediante ensayo de pull-out, la resistencia residual de la unión adhesiva.

Se han tomado como referencias la UNE-EN 1348 (7) y la Guía DITE $\mathrm{n}^{\circ}$ 001-5 (8) para el procedimiento de los ciclos de hielo y deshielo, en el que se han recogido tres muestras de discos cerámicos con la varilla roscada y la unión adhesiva correspondiente y se ha procedido a una inspección visual para detectar posibles defectos iniciales. Posteriormente se han ejecutado 15 ciclos de hielo/deshielo. En cada ciclo se han introducido las muestras en una cámara frigorífica a $-20^{\circ} \mathrm{C}$ durante 14 horas pasadas las cuales se han extraído de la cámara y dejado a temperatura ambiente durante 7 horas. Se han realizado observaciones visuales de las muestras y se ha iniciado de nuevo el ciclo. Finalizados los ciclos, a temperatura ambiente, se ha vertido anilina en la zona cerámica de sujeción de la varilla roscada y se ha observado si existía penetración del producto que revelara la presencia de fisuras (Figura 14).

Una vez comprobado que en ninguna de las tres piezas se detectaban fisuras, se ha evaluado su resistencia residual con ensayos pull-out a temperatura ambiente siguiendo el mismo procedimiento que en el punto 3.2, con carga aplicada mediante control de desplazamiento a una velocidad de $1,25 \mathrm{~mm} /$ min. Vistos los resultados comparados con los realizados en el apartado 3.2, se ha concluido que los ciclos de hielo y deshielo no producían fisuras en las piezas ni reducían su capacidad portante.

Entonces se ha evaluado la unión adhesiva con ciclos calor/ frío para provocar choque térmico, usando como referencia la normativa UNE-EN 12003 (9), en los que se ha sometido cada probeta a cuatro ciclos de cambio brusco de temperatura de 23 a $100^{\circ} \mathrm{C}$, sumergiéndolas en agua a dicha temperatura durante 5 minutos. Después del último ciclo se ha dejado enfriar a temperatura ambiente y se ha vuelto a utilizar anilina para revelar la existencia de posibles fisuras en las piezas. Tampoco en este caso se han detectado. $\mathrm{Y}$ al volver a evaluar su resistencia residual con ensayos pull-out y comparar sus resultados con los ensayados en condiciones normales (punto 3.2) tampoco se ha advertido reducción de su resistencia portante.

\section{CONCLUSIONES DE LA CAMPAÑA DE ENSAYOS}

Mediante los ensayos realizados se han estudiado los requerimientos mecánicos (resistencia y estabilidad) y los de durabilidad (ambiental y fatigas) del sistema Materia CAB Disset para el Centro de Arte Botín. 

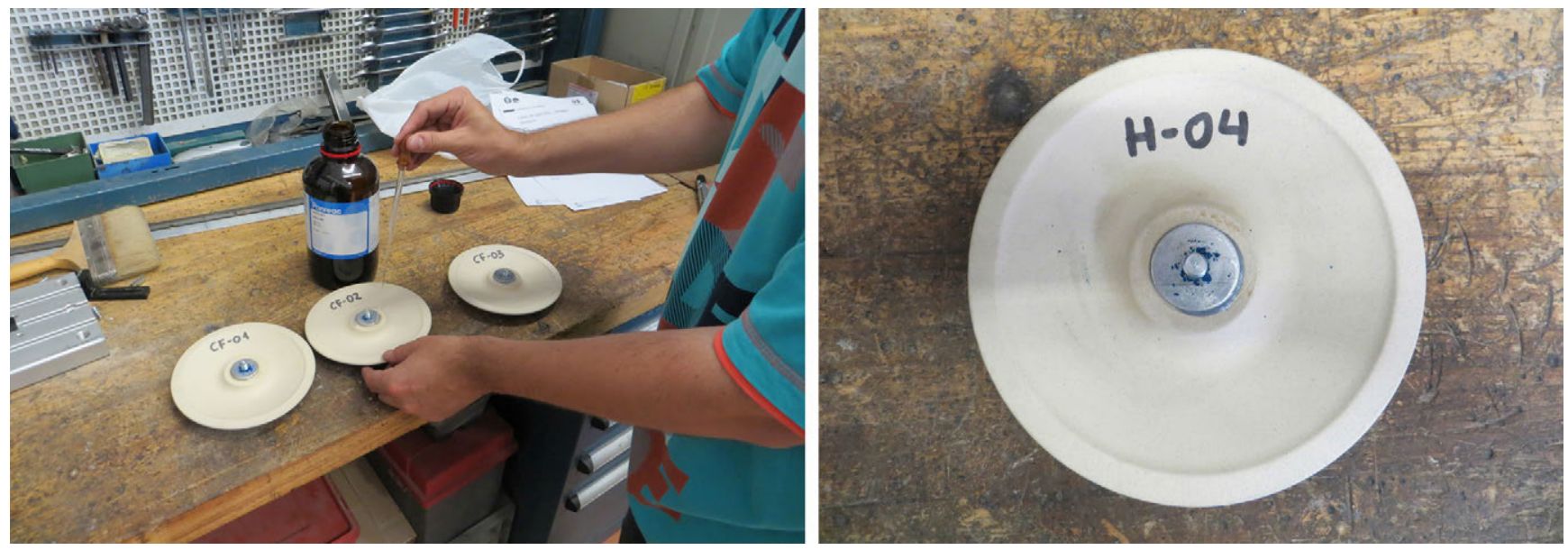

Figura 14. Aplicación de anilina en las muestras y detalle sin presencia de fisuras (Archivo LITEM).

En el ámbito de los requisitos mecánicos se han ejecutado ensayos de arrancamiento (pull-out) tanto entre el tornillo de sujeción y la placa de aluminio como entre éste y la pieza cerámica que demuestran un comportamiento sobradamente aceptable de estos tres elementos ante cargas muy superiores a las de servicio. Incluso no han mostrado afectación en condiciones de temperatura extrema (con probetas calentadas hasta $70^{\circ} \mathrm{C}$ ) y en envejecimiento acelerado que han dado valores promedio de $3.306 \mathrm{~N}$ en el arranque de los tornillos de las bandejas de aluminio y valores promedio de $3.324 \mathrm{~N}$ en el arranque de las varillas roscadas de las piezas cerámicas, ambos valores muy por encima de los que deben soportar.

En el ámbito de los requisitos de durabilidad se han realizado ensayos de comportamiento ante ambientes agresivos o extremos. Tanto la bandeja de aluminio como la unión de las piezas cerámicas con las varillas roscadas se han sometido a un proceso de envejecimiento acelerado en cámara de niebla salina y los resultados han verificado que no se ha producido una variación relevante en las propiedades de estos materiales, sin corrosiones aparentes (sólo cambios de tonalidad en las superficies metálicas) ni pérdidas de capacidad portante.
Dentro de los ensayos de durabilidad también se ha sometido a ciclos de hielo/deshielo y choque térmico a la unión del tornillo con la pieza cerámica para comprobar si hay afectación en la capacidad adhesiva de la resina que los fija. También en este caso no se han producido ni fisuras en las piezas cerámicas ni pérdida de capacidad portante de las resinas.

Por todo ello se ha concluido que este novedoso sistema de envolvente para el Centro de Arte Botín resulta viable y fiable técnicamente para los requerimientos mecánicos y de durabilidad propuestos.

\section{AGRADECIMIENTOS}

Los autores quieren agradecer la generosa disposición de la empresa Disset, de su oficina técnica y de su director de marketing y estudios Bernat Cadafalch, que han facilitado las fuentes gráficas y fotográficas de este artículo, y a su director Juli Febrero, que ha alentado tanto este escrito como el monográfico en el que está contenido. También agradecer sus aportaciones en los ensayos realizados al LITEM (Laboratorio para la Innovación Tecnológica de Estructuras y Materiales) de la Universitat Politècnica de Catalunya en la Escuela de Ingenieros de Terrassa.

\section{REFERENCIAS}

(1) Piano, R. (2015). Centro Botín de Arte y Cultura, in Detail. AV Proyectos (71): 49-67.

(2) Araújo, R. (2007). La arquitectura como técnica (pp. 122-123). Madrid: ATC Ediciones, S. L.

(3) Fernández-Alonso, R. (2013). Escuela Universitaria de Magisterio en Granada. AV Monografías (159-160): 60-62.

(4) AAVV. (2007). Construccions Patents. New architecture made in Catalonia (pp. 90-93). Barcelona: Ed. Actar, Institut Ramon Llull y Deutsches Architekturmuseum.

(5) Bechthold, M., Kane, A., King, N. (2016). Ceramic Material Systems (pp. 74-77). Basilea: Ed. Birkhäuser.

(6) AENOR-CEN. (2012). UNE-EN ISO 9227:2012 Ensayos de corrosión en atmósferas artificiales. Ensayos de niebla salina. Asociación Española de Normalización (AENOR).

(7) AENOR-CEN. (2008). UNE-EN 1348:2008 Determinación de la resistencia a tracción de adhesivos cementosos. Asociación Española de Normalización (AENOR).

(8) EOTA. (2008). Guía DITE n. ${ }^{\circ}$ oo1-5 Anclajes metálicos para hormigón. Parte 5: Anclajes químicos. Organización para la Idoneidad Técnica Europea.

(9) AENOR-CEN. (1997). UNE-EN 12003:1997 Adhesivos para baldosas cerámicas. Determinación de la resistencia a la cizalladura de los adhesivos de resinas reactivas. Asociación Española de Normalización (AENOR). 